\title{
US Definitions, Current Use, and FDA Stance on Use of Platelet-Rich Plasma in Sports Medicine
}

\author{
Knut Beitzel, MA, MD ${ }^{1}$ Donald Allen, MD $^{2}$ John Apostolakos, BA ${ }^{2}$ Ryan P. Russell, MA ${ }^{2}$ \\ Mary Beth McCarthy, BS ${ }^{2}$ Gregory J. Gallo, $\mathrm{PhD}^{2}$ Mark P. Cote, PT, DPT, MSCTR ${ }^{2}$ \\ Augustus D. Mazzocca, MS, MD²
}

${ }^{1}$ Department of Orthopaedic Sports Medicine, Technical University

Munich, Munich, Germany

2 Department of Orthopaedic Surgery, University of Connecticut

Health Center, Farmington, Connecticut

Address for correspondence Augustus D. Mazzocca, MS, MD, Department of Orthopaedic Surgery, University of Connecticut, Farmington, CT 06034 (e-mail: mazzocca@uchc.edu).

J Knee Surg 2015;28:29-34.

\begin{abstract}
Keywords

- platelet-rich plasma

- PRP

- regulations

- FDA

With increased utilization of platelet-rich plasma (PRP), it is important for clinicians to understand the United States, the Food and Drug Administration (FDA) regulatory role and stance on PRP. Blood products such as PRP fall under the prevue of FDA's Center for Biologics Evaluation and Research (CBER). CBER is responsible for regulating human cells, tissues, and cellular and tissue-based products. The regulatory process for these products is described in the FDA's 21 CFR 1271 of the Code of Regulations. Under these regulations, certain products including blood products such as PRP are exempt and therefore do not follow the FDA's traditional regulatory pathway that includes animal studies and clinical trials. The $510(\mathrm{k})$ application is the pathway used to bring PRP preparation systems to the market. The $510(\mathrm{k})$ application allows devices that are "substantially equivalent" to a currently marketed device to come to the market. There are numerous PRP preparation systems on the market today with FDA clearance; however, nearly all of these systems have $510(\mathrm{k})$ clearance for producing platelet-rich preparations intended to be used to mix with bone graft materials to enhance bone graft handling properties in orthopedic practices. The use of PRP outside this setting, for example, an office injection, would be considered "off label." Clinicians are free to use a product off-label as long as certain responsibilities are met. Per CBER, when the intent is the practice of medicine, clinicians "have the responsibility to be well informed about the product, to base its use on firm scientific rationale and on sound medical evidence, and to maintain records of the product's use and effects." Finally, despite PRP being exempted, the language in 21 CFR 1271 has caused some recent concern over activated PRP; however to date, the FDA has not attempted to regulate activated PRP. Clinicians using activated PRP should be mindful of these concerns and continued to stay informed.
\end{abstract}

Platelet-rich preparations, especially platelet-rich plasma (PRP), have become an increasingly popular treatment for various musculoskeletal injuries. PRP is somewhat unique in that both the device used to concentrate platelets and the subsequent use of the PRP product fall under the purview of the United States Food and Drug Administration (FDA). With increased utilization of PRP, it is important for clinicians to understand the FDA's regulatory role and stance on PRP. This can be challenging as the various methods of obtaining and processing PRP fall under different areas of FDA regulations. received

June 2, 2014

accepted

July 24, 2014

published online

September 30, 2014
Copyright $\odot 2015$ by Thieme Medical Publishers, Inc., 333 Seventh Avenue, New York, NY 10001, USA. Tel: +1(212) 584-4662.
DOI http://dx.doi.org/ 10.1055/s-0034-1390030. ISSN 1538-8506. 
The purpose of this article is to provide meaningful information to common points of confusion regarding the FDA and the use of PRP. A common clinical scenario is presented in an effort to convey the information in a clinically relevant manner.

\section{Clinical Scenario: The Young Patient with Glenohumeral Arthritis}

A 40-year-old manual laborer comes into the office complaining of shoulder pain and dysfunction. Plain radiographs are consistent with advance glenohumeral arthritis and magnetic resonance imaging reveals an intact rotator cuff. The patient has failed conservative management consisting of nonsteroidal anti-inflammatory drugs and formal physical therapy. Current treatment options for this patient include an arthroplasty procedure (hemi vs. total) and an intra-articular injection. Total shoulder replacement has been shown to provide pain relief in young adults, however, 10 -year survival rates are low. ${ }^{1}$ Hemiarthroplasty in a young patient provides less predictable pain relief compared with total shoulder arthroplasty and may lead to increased glenoid erosion postoperatively. ${ }^{2}$ A corticosteroid injection may provide short-term relief, however multiple injections raises concern over damage to the rotator cuff. ${ }^{3-5}$

In these situations, the lack of a clear treatment choice often prompts an extended conversation regarding alternative treatment options. In most cases, the goal of treatment is to adequately manage the patient's symptoms to allow a shoulder replacement to be delayed as long as possible. In this regard, PRP may be a viable treatment option. Although there are no studies to support the use PRP in the treatment of glenohumeral arthritis, it has been used with some success in treating arthritis of the knee. ${ }^{6,7}$ Additionally, its benign nature as an autologous substance $^{8}$ and its potential from a biological perspective to reduce proinflammatory mediator production ${ }^{9,10}$ make it an attractive treatment option compared with corticosteroid injections or an arthroplasty procedure.

Following this conversation, the patient decides to try PRP and schedules a follow-up visit for an intra-articular injection. Before the patient's return visit, numerous questions regarding the FDA and PRP surface. Is it the device or the PRP that is approved? How does the FDA regulate PRP? What does the language in 21 CFR 1271 of the Code of Regulations mean in terms of use of PRP?

\section{Is It Platelet-Rich Plasma or the Platelet-Rich Plasma Device that Is Approved?}

The first point of confusion is in regards to the FDA approval of PRP devices. There are many pathways in which a drug, biologic, or medical device can be brought to the market. The regulatory process in regards to the application of biologics in orthopedics is outlined in a recent review article by Anz et al. ${ }^{11}$ In general, drugs are approved via New Drug Applications (NDA) or Abbreviated New Drug Applications, utilizing clinical data obtained from an Investigational New Drug (IND) application. Depending upon the type or classifi- cation, biologics may be approved through NDAs or Biologics License Applications (BLA), or cleared via Premarket Notification $(510(k))$. NDA and BLAs require clinical data obtained from an IND or Investigational Device Exemption (IDE) application; a 510(k) may not require clinical data. Depending upon risk classification, medical devices may either be approved through Premarket Approval (PMA) or a 510(k) clearance. PMAs will require clinical data obtained from an IDE application; a 510(k) may not require the clinical data.

The $510(\mathrm{k})$ application is for devices that are considered lower risk devices, and determined to be "substantially equivalent" to a previously cleared device. A device under this application is given a $510(\mathrm{k})$ clearance. ${ }^{12,13}$ An approved device under this application is given a $510(\mathrm{k})$ clearance. The term "clearance" rather than "approval" is used to note that the device is similar to an existing device, or predicate, and the clearance is limited to the indications of the predicate device. The $510(\mathrm{k})$ application is the pathway typically used to bring PRP preparation systems to the market. While the $510(\mathrm{k})$ clearance is important as it allows clinicians access to PRP preparation systems, it can be confusing as to what the clearance actually signifies. In contrast to the IND, IDE, and PMAs, $510(\mathrm{k})$ applications focus primarily on the safety of a device as well as its performance characteristics and typically do not require supporting clinical data. In its simplest form, a device with $510(\mathrm{k})$ clearance is considered to be substantially equivalent to a predicate device for a specific indication. ${ }^{12,13}$

There are numerous PRP preparation systems on the market today with FDA clearance; however, nearly all of these systems have $510(\mathrm{k})$ clearance for producing platelet-rich preparations intended to be mixed with bone graft materials to enhance bone graft handling properties in orthopedic practices. They are not intended for direct patient applications, such as injection or implant without prior mixing with the bone graft materials. The clearance applies only to the device and its intended use in an operative setting and makes no claim to its effectiveness for a particular indication. This clearance limits the marketing of the device to the specific indication for which it was cleared, but it does not restrict or limit the practice of medicine by the clinician. Specifically, a PRP system with $510(\mathrm{k})$ clearance indicates that the device is (1) safe in that it does not create a platelet preparation that is hazardous or dangerous and (2) that its performance is substantially equivalent to a predicate PRP device meaning it has demonstrated the capability to isolate the platelets from whole blood. The $510(\mathrm{k})$ clearance applies to producing PRP for use with bone graft materials to enhance bone graft handling properties. In addition, $510(\mathrm{k})$ clearance is not synonymous with approval for a specific indication. Product approval for a given indication requires a BLA or PMA application, which is a more rigorous and clinically dependent process, centered on establishing safety and efficacy. ${ }^{12}$

\section{By Definition, Platelet-Rich Plasma Is a Biologic}

Per their Web site, "the FDA is responsible for protecting the public health by regulating human and animal drugs, 
biologics (e.g. vaccines and cellular and gene therapies), medical devices, food and animal feed, cosmetics, and products that emit radiation." ${ }^{14}$ It is natural to ask where does PRP fall among these items. In its simplest form, the term "Platelet-Rich Plasma" refers to a sample of autologous plasma with platelet concentrations above baseline values. ${ }^{15-17}$ Under this definition, PRP is considered a biologic. Biologics are regulated by the FDA's CBER.

\section{How Is a Biologic Such as Platelet-Rich Plasma Regulated?}

For clinicians using PRP, it is important to understand how agencies such as CBER approach regulating a biologic. The first consideration is whether the PRP is being used as intended. As previously discussed, PRP devices are cleared for use in an operative setting to mix with bone graft materials to enhance bone graft handling properties. The use of PRP outside this setting, for example, an office injection, would be considered "off label." The term "off label" may conjure feelings of fright and alarm among clinicians. Offlabel use simply refers to the use of a device for an indication for which that device was not cleared or approved. Given that preparation systems come to market via the $510(\mathrm{k})$ clearance pathway, where the cleared devices are substantially equivalent to predicate devices for producing platelet-rich preparations intended for use in an operative setting, office injections are considered off label. ${ }^{18}$ For office injections to be on label and approved, a BLA or PMA would need to be submitted and PMA with animal studies and clinical trials (involving an IND or IDE) would be required. This is a time consuming and costly process that would require substantial funding and with the simpler and quicker 510(k) pathway in place, PRP will likely continue to be labeled for operative use.

It is important to note that the FDA does not regulate the practice of medicine. Clinicians are free to use a product off label as long as certain responsibilities are met. Per CBER, when the intent is the practice of medicine, clinicians "have the responsibility to be well informed about the product, to base its use on firm scientific rationale and on sound medical evidence, and to maintain records of the product's use and effects," however, they do not require submission of an IND or IDE or oversight from an institutional review board (IRB). ${ }^{19}$ Returning to our clinical scenario, the young patient with glenohumeral arthritis, a review of the literature is performed before their return visit to ensure the responsibilities set forth by CBER are met. There are several cell-based studies that have demonstrated positive effects of plasma preparations on human tendon, muscle, and bone tissue. ${ }^{8}$ While clinical studies have shown mixed results in terms of efficacy, PRP has been successfully administered in these studies without an apparent increase in adverse events. In consideration of the patient's problem and the lack of a clear treatment choice, along with positive laboratory data and supporting safety data, off-label use of PRP would seem to be warranted as it may have some potential benefit with little risk in comparison to an office injection of corticosteroids or the undertaking of an arthroplasty procedure.
Conversely, for clinician researchers who intended to use PRP in an investigational manner to determine safety and efficacy, a formal review including IND or IDE and IRB approval is required. However, per 21 CFR 312.2(b)(1), there are specific situations in which an IND application may not be required. In short, this criteria pertain to investigations where there is no intention to support a new indication, labeling, or significant change in advertising, and does not involve increasing risk to the subject. ${ }^{19}$ The study may be conducted in compliance with an IRB and within the promotion of the product, and it does not invoke 21 CFR 50.24, which pertains to exemption from informed consent for emergency research. Researchers interested in clinical investigations of PRP are encouraged to contact CBER and the FDA for more information. ${ }^{19}$

\section{Food and Drug Administration Code of Regulations: 21 CFR 1271}

At the center of confusion regarding the FDA's stance on PRP is 21 CFR 1271 of the Code of Regulations. In 1997, the FDA released a tiered risk-based approach to the regulation of human cells, tissues, cellular and tissue-based products (HCT/Ps) intended for implantation, transplantation, infusion, or transfer to a human recipient in Title 21, Part 1271 of the Code of Federal Regulations. ${ }^{20,21}$ Specifically, the FDA defined two categories of HCT/Ps: lower risk products, which are less than minimally manipulated, advertised for homologous use only, not intended to be combined with another product with the exception of simple electrolyte, and preservation solutions, which are either autologous or have a nonsystemic effect. HCT/Ps meeting these requirements are regulated under section 361 of the Public Health Service Act and are often referred to as 361 products. These products do not require PMA before coming to the market. Products that do not meet the earlier-mentioned criteria are considered high risk and are regulated under section 351 and require animal and clinical data through the FDA's traditional PMA pathway. Cellular products excluded from regulation under 21 CFR 1271 include, but are not limited to, blood products, minimally manipulated bone marrow, and xenografts. ${ }^{20,21}$

In 2005, the FDA issued a ruling related to the manipulation HCT/Ps. ${ }^{21}$ Specifically, the FDA declared that any procedure in which human cells are manipulated for clinical use is subject to oversight. The cells or tissue can be either more than or less than "minimally manipulated." When the cells or tissue are more than minimally manipulated, they may be treated like a "drug" and require approval status under current regulations. ${ }^{21}$ This clarification has primarily impacted cellular therapies, specifically the use of cultured stem cells where the culturing process was deemed to have "more than minimally manipulated" the cells. ${ }^{22,23}$ This interpretation sparked debate over the FDA authority to regulate the practice of medicine with respect to the use of biologics. ${ }^{24,25}$ An extended example of the potential implications of a broad interpretation of the language of 1271 is the change that occurs with activated PRP. While PRP obtained from a $510(\mathrm{k})$ cleared device is unregulated, the activation of PRP with 
calcium and/or thrombin creates variability in the release of platelet growth factors and kinetic activity, prompting concern as to whether the FDA would consider this to be a "more than minimally manipulated" product. ${ }^{24}$ To date, this ruling has not resulted in the FDA attempting to regulate activated PRP suggesting that the concern regarding the language of 1271 likely reflects the ongoing debate over the FDA's role rather than their intent to regulate platelet-rich products.

\section{Our Approach to Platelet-Rich Plasma Treatments}

Before scheduling an office visit for injection, PRP treatment is discussed extensively with the patient. This includes a review of current research in which both cell-based and clinical studies are discussed. We make a concerted effort to deliver this information in an unbiased fashion to allow the patient to make an informed decision. We also note that there is no guarantee that the PRP injection will provide relief. The regulatory issues surrounding PRP treatments are also discussed. We describe the process of obtaining PRP, highlighting the fact that the devices used are cleared for safe and effective processing of PRP. In addition, we encourage patients to discuss PRP with their family and primary care physician. If a patient elects for a PRP injection, outcome and qualitybased information is collected. All information is collected on a quality assurance sheet to document the reason for seeking injection, adverse events, and immediate outcome. In the days following the injection, a call is made to the patient to find out how they are doing and whether they have experienced any adverse or unexpected events. This process helps ensure CBER's requirement of records regarding the use, and the effects of PRP is maintained. To date, we have not observed an adverse event or unanticipated effect that falls outside the scope of what is normally expected from an office injection.

\section{Summary of the FDA Stance on Platelet-Rich Plasma}

- Currently, there are several systems for obtaining PRP that have received $510(\mathrm{k})$ clearances which indicates that a given preparation system is substantial equivalent to a predicate device in that it is safe and capable of producing PRP.

- Per the FDA, PRP's intended use is in an operative setting to mix with bone graft materials to enhance bone graft handling properties. Use outside this setting is considered to be off label.

- Clinicians using PRP off label (office injections) as part of good medical practice and in the best interest of the patient have the responsibility to be well informed on the product and to keep records of its use; however, they do not require oversight from the FDA.

- The language in 21 CFR 1271 regarding the manipulation of cells has impacted the use of cultured stem cells, causing concern for some over activated PRP. To date, the FDA has not attempted to regulate activated PRP. Clinicians using activated PRP should be mindful of these concerns and continued to stay informed.

\section{Funding}

The University of Connecticut Health Center/New England Musculoskeletal Institute has received direct funding and material support for this study from Arthrex, Inc. (Naples, FL). The company had no influence on study design, data collection, and interpretation of the results or the writing of the final manuscript.

\section{References}

1 Denard PJ, Raiss P, Sowa B, Walch G. Mid- to long-term follow-up of total shoulder arthroplasty using a keeled glenoid in young adults with primary glenohumeral arthritis. J Shoulder Elbow Surg 2013; 22(7):894-900

2 Denard PJ, Wirth MA, Orfaly RM. Management of glenohumeral arthritis in the young adult. J Bone Joint Surg Am 2011;93(9): 885-892

3 Carofino B, Chowaniec DM, McCarthy MB, et al. Corticosteroids and local anesthetics decrease positive effects of platelet-rich plasma: an in vitro study on human tendon cells. Arthroscopy 2012;28(5):711-719

4 Wong MW, Tang YY, Lee SK, Fu BS. Glucocorticoids suppress proteoglycan production by human tenocytes. Acta Orthop 2005;76(6):927-931

5 Wong MW, Tang YY, Lee SK, Fu BS, Chan BP, Chan CK. Effect of dexamethasone on cultured human tenocytes and its reversibility by platelet-derived growth factor. J Bone Joint Surg Am 2003;85A(10):1914-1920

6 Gobbi A, Lad D, Karnatzikos G. The effects of repeated intraarticular PRP injections on clinical outcomes of early osteoarthritis of the knee. Knee Surg Sports Traumatol Arthrosc 2014

7 Chang KV, Hung CY, Aliwarga F, Wang TG, Han DS, Chen WS. Comparative effectiveness of platelet-rich plasma injections for treating knee joint cartilage degenerative pathology: a systematic review and meta-analysis. Arch Phys Med Rehabil 2014;95(3): 562-575

8 Mazzocca AD, McCarthy MB, Chowaniec DM, et al. The positive effects of different platelet-rich plasma methods on human muscle, bone, and tendon cells. Am J Sports Med 2012;40(8): 1742-1749

9 Braun HJ, Kim HJ, Chu CR, Dragoo JL. The effect of platelet-rich plasma formulations and blood products on human synoviocytes: implications for intra-articular injury and therapy. Am J Sports Med 2014;42(5):1204-1210

10 Browning SR, Weiser AM, Woolf N, et al. Platelet-rich plasma increases matrix metalloproteinases in cultures of human synovial fibroblasts. J Bone Joint Surg Am 2012;94(23):e1721-e1727

11 Anz AW, Hackel JG, Nilssen EC, Andrews JR. Application of biologics in the treatment of the rotator cuff, meniscus, cartilage, and osteoarthritis. J Am Acad Orthop Surg 2014;22(2):68-79

12 Sutton B. Overview of regulatory requirements: medical devices. Available at: http://www.fda.gov/downloads/Training/CDRHLearn/ UCM400786.pdf. Accessed May 6, 2014

13 Rosecrans H. 510(k) Overview. Available at: http://www.fda.gov/ downloads/Training/CDRHLearn/UCM391459.pdf. Accessed May 6, 2014

14 U.S. Food and Drug Administration. Is it really FDA approved? Available at: http://www.fda.gov/ForConsumers/ConsumerUpdates/ucm047470.htm. Accessed April 30, 2014

15 Russell RP, Apostolakos J, Hirose T, Cote MP, Mazzocca AD. Variability of platelet-rich plasma preparations. Sports Med Arthrosc 2013;21(4):186-190 
16 Mazzocca AD, McCarthy MB, Chowaniec DM, et al. Platelet-rich plasma differs according to preparation method and human variability. J Bone Joint Surg Am 2012;94(4):308-316

17 Marques LF, Stessuk T, Camargo IC, Sabeh Junior N, Santos LD, Ribeiro-Paes JT. Platelet-rich plasma (PRP): methodological aspects and clinical applications. Platelets 2014; February 10 (Epub ahead of print); doi: 10.3109/09537104.2014.881991

18 Fan Y. An Overview of Medical Device Regulations in the US. ISCT North America Regional Meeting; September 8, 2013

19 U.S. Food and Drug Administration. "Off-Label" and Investigational Use of Marketed Drugs, Biologics, and Medical Devices - Information Sheet. Available at: http://www.fda.gov/RegulatoryInformation/Guidances/ucm126486.htm. Accessed April 30, 2014

20 US Department of Health and Human Services. Human cells, tissues, and cellular and tissue-based products. 21 CFR Part 1271. Available at: http://www.accessdata.fda.gov/scripts/cdrh/ cfdocs/cfcfr/CFRSearch.cfm?CFRPart=1271\&showFR=1. Accessed April 30, 2014
21 Barone SB. "361" Human Cells, Tissues, and Cellular and TissueBased Products (HCT/Ps). The Office of Cellular, Tissue, and Gene Therapies Web Seminar Series. Available at: http://wwwfdagov/ downloads/BiologicsBloodVaccines/NewsEvents/UCM251330ppt. Accessed April 30, 2014

22 United States District Court for the District of Columbia Civil Action No. 10-1327. United States of America versus Regenerative Sciences, LLC. Available at: https://ecf.dcd.uscourts.gov/cgi-bin/ show_public_doc?2010cv1327-47. Accessed April 30, 2014

23 Malarkey MA. Warning letter to IntelliCell Biosciences, Inc: March 13, 2012. Available at: http://www.fda.gov/ICECI/EnforcementActions/ WarningLetters/2012/ucm297245.htm. Accessed April 30, 2014

24 Centeno CJ, Fuerst M, Faulkner SJ, Freeman M. Is cosmetic plateletrich plasma a drug to be regulated by the Food and Drug Administration? J Cosmet Dermatol 2011;10(3):171-173

25 Freeman MD, Fuerst MS. Does the FDA have regulatory authority over adult autologous stem cell therapies? 21 CFR 1271 and the emperor's new clothes. J Transl Med 2012;10(1):60 